\title{
Application of sequential cyclic compression on cancer cells in a flexible microdevice
}

\author{
Sevgi Onal ${ }^{1,2^{*}}$, Maan M. Alkaisi ${ }^{1,2}$, and Volker $\operatorname{Nock}^{1,2,3^{*}}$ \\ 1 Electrical and Computer Engineering, University of Canterbury, Christchurch, New \\ Zealand \\ 2 MacDiarmid Institute for Advanced Materials and Nanotechnology, Wellington, New \\ Zealand \\ 3 Biomolecular Interaction Centre, University of Canterbury, Christchurch, New Zealand \\ * sevgi.onal@pg.canterbury.ac.nz and volker.nock@canterbury.ac.nz
}

\begin{abstract}
Mechanical forces shape physiological structure and function within cell and tissue microenvironments, during which cells strive to restore their shape or develop an adaptive mechanism to maintain cell integrity depending on strength and type of the mechanical loading. While some cells are shown to experience permanent plastic deformation after a repetitive mechanical tensile loading and unloading, the impact of such repetitive compression on plastic deformation of cells is yet to be discovered. As such, the ability to apply cyclic compression is crucial for any experimental setup aimed at the study of mechanical compression taking place in cell and tissue microenvironments. Here, the capability of our microfluidic compression platform to aid in the observation of the sequential cyclic compression of live cell actin is illustrated using SKOV-3 ovarian cancer cells. Live imaging of the actin cytoskeleton dynamics of the compressed cells was performed for the applied varying pressures in ascending order during cell compression. Additionally, recovery of the compressed cells was investigated by capturing actin cytoskeleton and nuclei profiles of the cells at zero time and 24 h-recovery after compression in end point assays. This was performed for a range of mild pressures within the physiological range. The extent of recovery of the compressed cells can give insights into the plasticity of the cancer cells by imaging cell membrane bulges and actin cytoskeleton and measuring the shape descriptors of cell nuclei. As demonstrated in this work, the developed platform can control the strength and duration of cyclic compression, while enabling the observation of morphological and cytoskeletal and nuclear changes in cells, thus providing a powerful new tool for the study of mechanobiological processes in cancer and cell biology.
\end{abstract}

\section{Introduction}

While some cells experience permanent plastic deformation after a repetitive mechanical tensile loading and unloading, the impact of such repetitive compression on plastic deformation of cells remains unknown [1,2. As such, the ability to apply cyclic compression is crucial for any experimental setup aimed at the study of mechanical compression taking place in cell and tissue microenvironments $3[5]$. For instance, in ovarian cancer, cells are exposed to chronic compressive stress from different sources such as tumour growth, displacement within stromal tissue and hydrostatic pressure out 
of ascitic fluid in peritoneal cavity 3, 6. Such compression is estimated to reach 18.9 $\mathrm{kPa}$ for human tumours and can exceed $20 \mathrm{kPa}$ based on the experimental data from murine tumours, while those values can be higher in situ with the impact of the surrounding extracellular matrix in tumour microenvironment 3 , 7. Mimicking chronic compression profiles and physiological pressure values in an in vitro dynamic compression system is necessary to further study impact of compressive stress in cancer.

In the presence of a mechanical stress, cells strive to restore their shape to maintain cell integrity after the removal of mechanical loading. However, cells may show a plastic response in the form of cytoskeletal bond ruptures and an incomplete cell shape recovery [1]. Meanwhile, cells produce an adaptive mechanism via these bond ruptures to reduce the mechanical cell stress and thus protect themselves against mechanical damage, while subsequently deforming under the mechanical load [1,8. Such cell response and adaptive mechanism have been shown by Bonakdar et al. for cyclic mechanical tensile loading [1], but comparable investigations into compressive loading remain outstanding. In particular in relation to cancer, an urgent need exists to investigate the cell deformation and recovery as response to cyclic mechanical compressive loading on living cells.

Asem et al. have recently shown that human peritoneal mesothelial cells drastically change their morphology under compression, as evidenced by formation of sub-micrometer scale intercellular projections with changes in the actin cytoskeleton and a resulting more mesenchymal phenotype. Depending on the compression strength and time length, such as $3 \mathrm{kPa}$ for 24-hour, mesothelial cells were observed to exhibit modified nanoscale cell surface projections and form actin-based tunneling nanotubes (TNTs) used to physically interact with ovarian cancer cells [9].

In general, cells under compression display a change in cell height and thus cell deformation in vertical direction. The nuclei region, as the highest cell compartment, contributes most to the overall cell height. Thus, when cells are compressed, the nuclei and actin cytoskeleton components supporting the cell body are highly mechanoresponsive to the applied compression. Ho et al. has performed volumetric scanning of the single cells being flatten and observed such a decrease in cell height as per increasing pressures applied externally through a compression control valve [2].

In itself, the nucleus is a mechanosensitive organelle that can respond to solid stress as we have recently shown by investigating nuclear deformations in ovarian cancer cells exposed to compression at increasing applied pressures [5]. Solid stress-induced nuclear deformations can change the activity of nuclear pore complexes and associated proteins. Such nuclear perturbations in turn modulate the nuclear import of transcription factors, which can alter gene expression and induction of DNA repair programs 10.

With the purpose of applying compression in a cyclic, dynamic, and controlled manner for the investigation of cell deformation and recovery, we have developed a robust cyclic compression microfluidic method based on a flexible microdevice, which provides extensive control of the amount, duration, and mode within a pressure profile. Fabrication and characterization of the multilayer microfluidic platform, the observation of directional growth of cells and mechanical cell lysis as an end point assay under static state and highly pressurized state within this platform were demonstrated previously 4,5 .

In this paper we present the use of the microfluidic platform for the in vitro application of cyclic cell compression to mimic biologically relevant compression profiles occurring in cellular microenvironments. In particular, we demonstrate applicability of the platform for the chronic exposure of ovarian cancer cells to compressive stress. As such, the current work extends the use of the platform to the application of cyclic compressions at and beyond physiological pressure values in a sequential fashion. We further apply this method to study dynamic biomechanical processes by recording 
GFP-tagged actin dynamics of live cells under compression. We also run end point assays of actin and nuclei deformations in compressed cells at zero time or at 24 h-recovery after compression. We demonstrate that the platform can control the strength and duration of cyclic compression, thus providing a powerful new tool for the study of mechanobiological processes.

\section{Materials and methods}

\subsection{Device fabrication and characterization}

Microdevice design, fabrication and characterization were explained in detail previously 5]. In brief, the multilayer flexible microdevice is composed of a control microchannel at the top, a polydimethylsiloxane (PDMS) membrane and monolithically attached micro-piston in the middle, suspended in a bottom microchannel enclosed by a glass substrate on which cells are cultured (Fig 1). The layers were fabricated out of PDMS via different soft lithography methods including standard replica molding for the control layer, spin-coating for the membrane/micro-piston layer, and exclusion molding for the bottom layer. Individual layers were assembled via alignment marks in each layer and the aligned PDMS construct was plasma-bonded to the glass. Dimensions of the device compartments were measured after each fabrication to ensure repeatability in predicting the external pressure amount via computational modelling. This ensured good control of piston contact pressures applied to cells on-chip during cell compression experiments.

\subsection{Computational model and experimental validation}

A previously developed finite element model (FEM) based on hyperelastic Saint Venant-Kirchhoff theory for actuation of the flexible microdevice 5] was implemented in COMSOL Multiphysics (V5.5, COMSOL) and used with different membrane thicknesses obtained from experimental thickness measurements of membranes within the fabricated devices. Vertical displacement and maximum piston contact pressure as per the applied external pressure were simulated and analysed.

\subsection{Cell culture, Matrigel coating and chip loading with cells}

SKOV-3 ovarian cancer cells (kindly supplied by the Laboratory for Cell and Protein Regulation at the University of Otago, Christchurch) were cultured in Earle's salts and L-glutamine-positive MEM (Gibco) supplemented with 10\% fetal bovine serum (FBS, Life Technologies), $1 \%$ of penicillin/streptomycin (Life Technologies), and $0.2 \%$ fungizone (Life Technologies) in a humidified atmosphere of $5 \% \mathrm{CO}_{2}$ at $37^{\circ} \mathrm{C}$. The cell channel of the flexible microdevice was coated with $100 \mu \mathrm{g} / \mathrm{ml}$ Matrigel (Corning, 356237) for 60-70 minutes before cell seeding (Fig 1(b)). $100 \mu \mathrm{g} / \mathrm{ml}$ Matrigel was used as it has been previously shown to favour cancer cell adhesion and mimic the extracellular matrix (ECM) microenvironment in comparison to other substrates [11. SKOV-3 cells at a seeding density of $1.5 \times 10^{6}$ cells $/ \mathrm{ml}$ were loaded using piston-retracted loading. In this method of chip loading with cells, the PDMS membrane with the attached micro-piston was retracted out of the culture channel towards the top control channel using an applied negative pressure of $615(-615)$ mbar to favour flow of seeding solution for homogenous distribution of cells. Seeded cells were cultured for 3-4 days on-chip until confluency S1 Fig). One day before experiments cell media in the channel was changed to $\mathrm{CO}_{2}$ independent medium (Gibco) supplemented with $10 \%$ fetal bovine serum, 2\% L-Glutamine (Gibco, $200 \mathrm{mM}$ ), $1 \%$ of 
a)

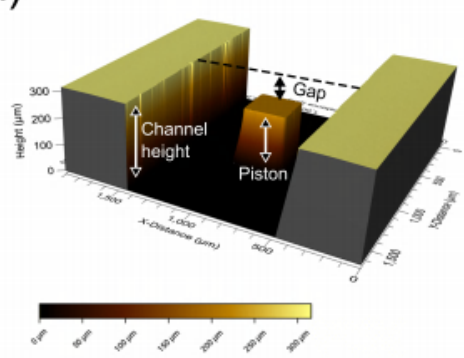

c)

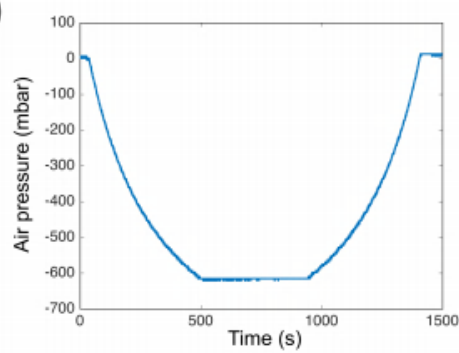

b)

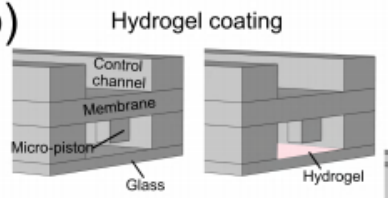

Cyclic compression

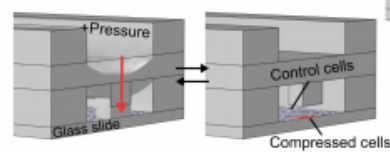

d)

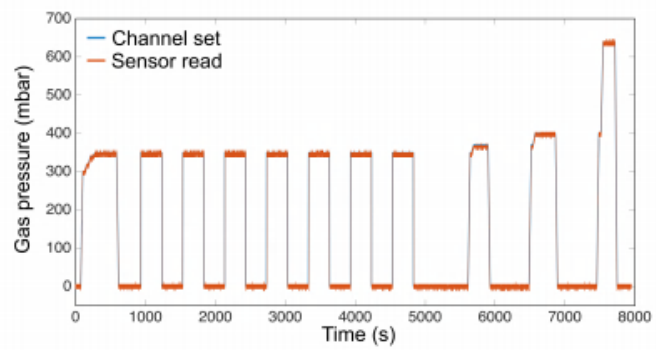

Fig 1. Device design, operation and applied pressure profiles. (a) 3D optical profilometer image of the micro-piston device showing the compartment including total height of the channel where the micro-piston is suspended, height of the micro-piston and the gap between the piston and surface of the channel. (b) Device operation steps from hydrogel coating, to piston-retracted loading of cells, and to cyclic compression on cells. Device was kept at static state during hydrogel loading. During piston-retracted loading, membrane, and thus micro-piston, were retracted from static position towards top control channel by the applied negative pressure and after cell-loading and settlement of the cells, flexible components were pumped down to their initial position. Cyclic compression on cells is illustrated by the membrane deflection and micro-piston brought onto the cells by the pressure applied through the control channel and retracted back after compression, which was applied repeatedly. Representative pressure profiles for piston-retracted loading (c), and sequential cyclic compression process (d).

penicillin/streptomycin, and $0.2 \%$ fungizone. Cells were cultured overnight in the humidified atmosphere of a $\mathrm{CO}_{2}$ free incubator at $37^{\circ} \mathrm{C}$ until live cell compression and imaging on a pre-warmed custom microscope stage (LEC060, LabIVF) at $37^{\circ} \mathrm{C}$.

\subsection{Device operation for sequential cyclic compression}

The flexible microdevices were operated with cyclic compression profiles in sequential manner. A pressure ladder with gradually increasing pressure was applied until the micro-piston reached the cells and started to apply contact pressure. The membrane, and thus micro-piston, were actuated with a pressure controller (OB1 Mk3+, Elveflow) coupled to pressure sensors (MSP4, 7 bar, Elveflow) and sensor reader (MSR, Elveflow) operated via Elveflow Smart Interface software (ESI, v3.04). This was used to set the $\mathrm{N}_{2}$ external gas pressure values required at each step while applying the cyclic pressure profiles in an automated manner. Pressure values in this work are given in mbar for externally applied pressures, as per calibrated settings of the pressure pump, pressure sensor and sensor reader, while $\mathrm{kPa}$ is used for the piston contact pressures, which are based on conversion from values computed using mechanical modelling. 


\subsection{Live imaging of GFP-tagged actin cytoskeleton of cells}

The actin cytoskeleton profile was quantified by comparing cancer cells with and without applied force. Both, an end point assay and live imaging of the actin cytoskeleton of cells transduced with CellLight ${ }^{\mathrm{TM}}$ Actin-GFP, BacMam 2.0 (Invitrogen) were used to investigate the dynamic biomechanical processes in cancer cells under compression. The use of baculovirus for the Actin-GFP transduction was conducted under Environmental Protection Authority (EPA, New Zealand) approval number of GMD101847. Cells were seeded on-chip at a density of $1.5 \times 10^{6}$ cells $/ \mathrm{ml}$ and cultured overnight, allowing sufficient time for cell adherence. Cells were then transduced on-chip for actin-GFP at 30 PPC (particle per cell) with CellLight ${ }^{\mathrm{TM}}$ particles. Following this, cells were incubated for an additional 3 days and the cell media containing the particles was replenished daily until the day of the compression application.

\subsection{Endpoint assay staining method}

After compression experiments, cells were fixed on-chip with $4 \%$ paraformaldehyde (Alfa Aesar) for $30 \mathrm{~min}$. The nuclei and actin cytoskeleton of the cells in the cell-culture channels of the micro-piston devices were stained using Hoechst 33342 (Thermo Scientific, $1 \mu \mathrm{g} / \mathrm{ml}$ ) and CF $488 \mathrm{~A}$ phalloidin (Biotium, 1:40) according to manufacturers' instructions, with on-chip dye incubation for $20 \mathrm{~min}$ and $1.5 \mathrm{~h}$, respectively. Stained nuclei and actin cytoskeleton of the fixed cells were captured with an inverted fluorescence microscope (Nikon Eclipse Ti) and Hamamatsu camera (ORCA-Flash4.0 V2).

\subsection{Imaging and data analysis}

Live imaging of cyclic compression was performed via time-lapse phase contrast imaging at a rate of 1 frame per $100 \mathrm{~ms}$. Fluorescence images for actin-GFP were acquired following each step of the sequential cyclic compression. Images were processed and analysed using Image $\mathrm{J}$ (Fiji) 12. Regions of interest (ROIs) were drawn around the periphery of the micro-pistons. Corrected total cell fluorescence (CTCF) [13] was calculated as follows:

$\mathrm{CTCF}=$ Integrated Density - (Area of micro-piston ROI x Mean fluorescence of background readings)

For the analysis of cell nuclear deformation, ImageJ was used to extract ROIs for the cell nuclear boundaries by analysing particles function applied on the threshold images with Hoechst epi-fluorescence signals. ROIs obtained from the Hoechst-stained cell nuclei in this way were confirmed using corresponding phase-contrast images for validation of the nuclei segmentation. Area, circularity, and aspect ratio of the cell nuclei were measured from the ROIs. Two-hundred-fold magnification images were used during the analysis of Hoechst-stained nuclei of control (non-compressed) cells in control regions (CRs) around the micro-piston and compressed cells under the micro-piston at zero time or 24 h-recovery after the compression.

Mann-Whitney two-tailed test was used to determine the statistical significance of cell nuclear deformations. Statistical significance was taken as $\mathrm{p}<0.05$. Data were represented as mean \pm standard error of mean (s.e.m.). 


\section{Results and Discussion}

\section{Comparison of the experimental and computational results in micro-piston device}

Mechanical compression contributes to shaping physiological structure and function within cell and tissue microenvironments, not only in tissues that have explicit biomechanical functions, such as vasculature, joints and muscles, but also tumours 14 . While mechanical compression on living cells has been applicable in bulk systems, controlled and dynamic compression can be achieved particularly in microfluidic settings, which is mimicking the in vivo conditions. In this regard, we have developed a robust dynamic compression microfluidic method based on a flexible microdevice to apply compression on cells cultured on hydrogel coatings, with extensive control of the amount, duration, and mode within a pressure profile (Fig 1). In the current work we extended the use of our flexible microdevice platform to the application of cyclic compression at and beyond physiological pressure values in a sequential fashion for investigation of cell deformation and recovery with changes in actin and nuclei profiles. Based on the experimental data obtained from optical profilometer measurements of actuated micro-pistons (Fig 1(a)), COMSOL simulations were performed to predict the piston contact pressure (Fig 2 and pistons were used to perform cyclic cell compression. Details of the sequential cyclic compression process at mild pressures, up to cell lysis at severe pressures, could be recorded and analysed, together with cell viability [15]. Computational modelling used to calculate the maximum piston contact pressure was further validated experimentally using independent microdevices with different membrane thicknesses to enhance reproducibility and repeatability of our flexible microdevice-based compression method. In our previous work we showed that simulations were in good agreement with optical profilometer measurements of vertical displacement of the PDMS membrane and thus micro-piston at given externally applied pressures [5]. Here, we extended this agreement to a correlation between the computational model and cell compression applications within independent micro-piston devices fabricated with different membrane thicknesses. For instance, when small variations formed in spin-coated PDMS membrane thicknesses, our model was able to predict what external pressures needed to be applied through the control microchannel to obtain comparable piston contact pressures on cells, as demonstrated in Fig 2 and Table 1 for thicknesses of 246, 253 and $258 \mu \mathrm{m}$ measured out of PDMS membrane coated on a 4-inch photoresist master. The model could also predict external pressure amounts that need to be applied when the same membrane thickness, such as $235 \mu \mathrm{m}$, was used to obtain different piston contact pressures on cells in independent microdevices (Table 1). Measurements demonstrated that the amount of pressure required to deflect each membrane to bring the micro-piston into contact with cells was in accordance with the computationally modelled external pressures (Fig 2(a)). Table 1 shows the compression applied to cancer cells via different membrane thicknesses for externally applied pressures and the resulting maximum piston contact pressures. Fig $2 \mathrm{~b}$ shows that comparable piston contact pressures were achieved by mildly compressing cells with applied external pressures, which were experimentally matching the pressure amounts in simulations $\left(\mathrm{R}^{2}=0.9967\right)$. This indicates that the model is robust across different membrane thicknesses and independent devices, and can be used to predict the piston contact pressure resulting from externally applied pressures.

While inferring these results, the cell compression process was imaged in detail in Matrigel-coated microchannels at a speed of 1 frame per $100 \mathrm{~ms}$, as shown in S1 Movie As illustrated by this time-lapse video, the platform allowed for the temporal evolution of the dynamic cell compression to be investigated in each cycle while the piston was contacting cells and applying compression. Distinct cell membrane bulges forming 


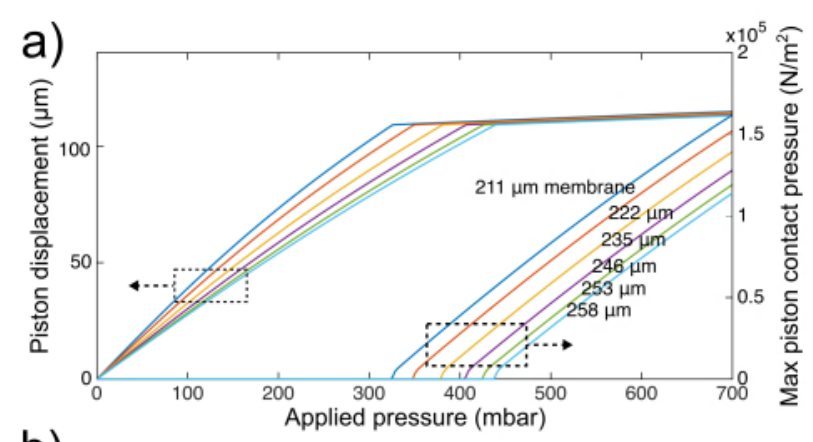

b)

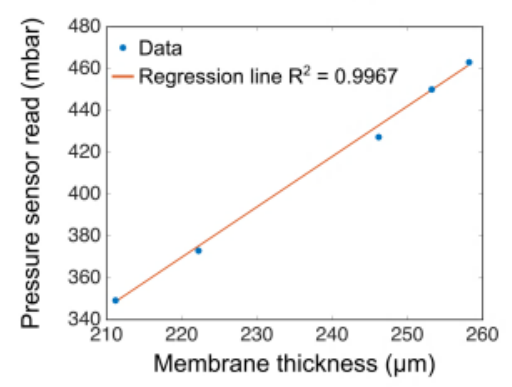

Fig 2. Computational and experimental validation of the micro-piston contact pressures across different membrane thicknesses. (a) Simulated piston displacement and maximum piston contact pressures at different membrane thicknesses as function of applied pressure. The left pattern of curves refers to the left y-axis (piston displacement $(\mu \mathrm{m})$ ), while the curve pattern on the right refers to the right y-axis (max piston contact pressure $\left(\mathrm{N} / \mathrm{m}^{2}\right)$ ), resulted at each externally applied pressure shown in x-axis. At a certain applied pressure (x-axis), micro-piston moves towards the bottom substrate and thus shows a displacement (left y-axis) depending on membrane thickness and applies a corresponding contact pressure (right y-axis). Colors of the curves refers to the membrane thicknesses indicated on the graph. (b) Correlation of independent experiments with the pressure sensor read for the externally applied pressure based on simulations run for the corresponding membrane thicknesses, resulting in a similar piston contact pressure.

Table 1. Compression applied to cancer cells via fabricated different membrane thicknesses, showing externally applied pressures with sensor readings out of experiments (in parentheses) and the predicted resulting internal maximum piston contact pressures.

\begin{tabular}{|c|l|c|}
\hline Membrane thickness (pm) & $\begin{array}{l}\text { Applied pressure } \\
\text { External (mbar) }\end{array}$ & $\begin{array}{c}\text { Piston contact pressure } \\
\text { Internal (kPa) }\end{array}$ \\
\hline 211 & $354(350.01)$ & 15.6 \\
\hline 222 & $380(373.74)$ & 15.9 \\
\hline 235 & $400(397.47)$ & 12.5 \\
\hline 235 & $410(409.34)$ & 17.9 \\
\hline 235 & $420(415.27)$ & 20.7 \\
\hline 246 & $430(427.94)$ & 15.1 \\
\hline 253 & $450(450.87)$ & 15.6 \\
\hline 258 & $465(463.61)$ & 15.6 \\
\hline 258 & $470(468.68)$ & 17.9 \\
\hline
\end{tabular}


during such compression at piston contact pressure of $15.6 \mathrm{kPa}$ were visible and recovery of cell membranes from these bulges could be observed when the piston was lifted off during rest stages. Although the device is capable of temporal control of cell compression (e.g. to decrease, increase or remove the pressure at any certain time interval), duration of each stage in a cycle was set to be 5 minutes (i.e. 5 -min compressed stage followed by 5 -min rest stage in each cycle). In this compression application with such an interval, cells were aimed to have enough time at each stage to complete their bulge formation or recovery, since this was considered as important for the investigation of cell deformation and recovery after compression, observed from cell behavior via detailed time-lapse imaging in S1 Movie. Furthermore, sensor readings in this video showed that the micro-piston actuated according to externally applied pressures with an extensive control and could be operated in short durations gradually or suddenly when needed. While compression frequencies in an in vivo tumor microenvironment are not fully known, such cyclic compression is also important for maintenance of cell culture during mechanical compressions in an in vitro experimental setup, enhancing media flow underneath micro-piston. Experimental dynamic compressions by others in literature include a frequency of $(0.1-30 \mathrm{~Hz})$ with an applied stress of $5.1,9.3,12.9$ and $18.7 \mathrm{kPa}$ on breast cancer cells for short duration $(30-300 \mathrm{~s})$ by Takao et al., where they observed a mixed mode of apoptosis and necrosis dominant mechanical cell death of compressed monolayers in a bulk compression platform [16]. On the other hand, Novak et al. used a frequency of $0.05 \mathrm{~Hz}$ in cyclic compression applied at $3.9-6.5 \mathrm{kPa}$ for 24 hours and observed an increase in proliferation capacity and decrease in apoptosis of OVCAR3 and OVSAHO ovarian cancer cells cultured in 3D hydrogel components in a bioreactor at millimeter scales 3. In another example, Ho et al. applied cyclic compression between the external pressures of 10 and 15 psi (68.9 and $103.4 \mathrm{kPa}$ ) at $0.5 \mathrm{~Hz}$ for 6 minutes. In a single cell microfluidic compression platform, their application resulted with full recovery and thus no permanent plastic deformation in MCF10A normal breast epithelial cells after compression 2]. Comparatively, as extensively demonstrated in the current work, micro-piston device was also used to apply cyclic compression with a low frequency in microfluidic settings to study cell compression, deformation and recovery after compression. The amount of the pressure, duration of the application and number of the cycles clearly affected the cell morphology and compression, as demonstrated and discussed further in the following.

\section{Live cell actin profiles of cancer cells during sequential cyclic compression}

We were further able to observe how the application of sequential cyclic compression lead to dynamic changes in GFP-tagged live cell actin when compressed at various applied pressures (see S2 Movie). For these experiments, SKOV-3 ovarian cancer cells were transduced on-chip with CellLight Actin-GFP, BacMam 2.0 baculovirus to visualize the dynamic biomechanical processes under compression (Fig 3). Specifically, the actin cytoskeleton profile was quantified for the cancer cell response at compressed and at rest stages during the compression process (Fig 4). For this, we dynamically stimulated and compressed the actin-GFP transduced cancer cells with various intermediate actuation pressures in a sequential cyclic manner following a sequence from Mild (15.6-15.9 kPa) to Intermediate 1 (23.8-26.8 kPa), to Intermediate 2 (37.8-41.7 $\mathrm{kPa}$ ) and Severe $(127.8-140 \mathrm{kPa})$ piston contact pressures. For the sequence shown in Fig 1(d), first, a pressure amount ladder was formed up to a pressure in the mild compression range, represented by an externally applied pressure of 350 mbar and resulting in a piston contact pressure of $15.6 \mathrm{kPa}$, during which the piston reached the cells at the bottom surface and cells started to deform distinctly. Then cyclic 
compression was applied at mild pressure for a total of 1 hour during which cells were compressed for $5 \mathrm{~min}$ at $15.6 \mathrm{kPa}$, followed alternately by a rest for 5 min at $0 \mathrm{kPa}$. To track cell response, actin-GFP signals of the compressed cells were captured before compression, as well as during the first and last cycles of the cyclic compression stage. At the end of this sequence, the externally applied pressure was sequentially increased first to $370 \mathrm{mbar}$, then to $400 \mathrm{mbar}$ and finally $640 \mathrm{mbar}$, resulting in the respective piston contact pressures of $23.8 \mathrm{kPa}$ (Intermediate 1), $37.8 \mathrm{kPa}$ (Intermediate 2) and $140 \mathrm{kPa}$ (Severe). During this stage, cells were compressed for up to 2 min at each higher pressure (Fig 1(d)) and actin-GFP signals of the compressed cells were captured (Fig 3). Observations were quantified based on CTCF and results are plotted as in Fig 4 A clear correlation between the actin-GFP signal of the cells compressed under the piston and the applied pressure could be observed, with the signal decreasing with increasing pressure. The results illustrate how the actin cytoskeleton of cancer cells changes in response to compressive stress under live-cell conditions. The change in actin signal was statistically different for compressed cells under micro-piston compared to non-compressed cells in control regions around micro-piston at all applied compression levels ( $\mathrm{p}<0.05$, see S1 File), where actin signal changes in the control regions remained statistically insignificant $(\mathrm{p}>0.05)$. While actin of most of the cells was disrupted for higher pressures at Intermediate 2 and Severe levels compared to initial stages of mild compression ( $\mathrm{p}<0.05$, see S1 File), a few cells remained resistant to the increasing pressure ranges, as evidenced by their retained fluorescent signal under micro-piston. $\mathrm{CTCF}$ at rest stage after upper mildly compression at Intermediate 2 level was statistically significantly different from the stage before compression $(\mathrm{p}=0.0229)$ and the rest stage after first cycle of mild compression $(\mathrm{p}=0.0352)$, which shows that Intermediate 2 level caused disruption of cell actin. This disruption was significantly higher at Severe level of applied pressures compared to other levels $(\mathrm{p}<0.05$, see $\mathrm{S} 1$ File). As such, these results expand on the work by Ho et al., who used a certain pressure range alternating between external pressures of 10 and 15 psi (68.9 and 103.4 $\mathrm{kPa}$ ) at $0.5 \mathrm{~Hz}$ applied for 6 min to study the mechanics of non-cancerous breast epithelial cell deformation by compression and observed formation of new actin stress fibers and thickening of a pre-existing actin structure in a very limited number of cells 2. In contrast, the current work observed live-cell actin in the cytoskeleton from deformation to ruptures at a wider range of applied pressures across several stages of the sequential cyclic compression.

The current device also allowed the response of individual cells at the interface between compressed and non-compressed regions in the periphery of the micro-piston to pe studied, thus providing a degree of control of the localization of compression (see S2 Movie). As depicted in the video, parts of cells at the interface were subject to compression, while the rest remained uncompressed. Thus, the cell actin was deformed and damaged only partially in the compressed part depending on the magnitude of the applied pressure, whereas it remained more intact at the non-compressed part.

\section{Actin cytoskeleton and nuclei profiles of cancer cells after compression and recovery}

Applicability of the cyclic compression on the platform was further demonstrated by recording the actin and nuclei deformation as an end point assay in cancer cells fixed at zero time or after 24 h-recovery following 1 hour-long cyclic compressions. To this end, we show progression of the compressed cells when live culture was maintained for 24 h-recovery after compression (S2 Fig). Nuclear shape analysis was performed on fluorescent images of control (non-compressed) and compressed cells at zero time or 24 h-recovery after compression S3 Fig, Fig 5 (a) and S4 Fig). No significant change 
bioRxiv preprint doi: https://doi.org/10.1101/2022.01.07.475458; this version posted January 10, 2022. The copyright holder for this preprint (which was not certified by peer review) is the author/funder. All rights reserved. No reuse allowed without permission.

a)

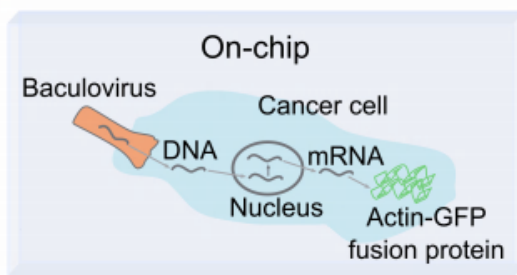

c)
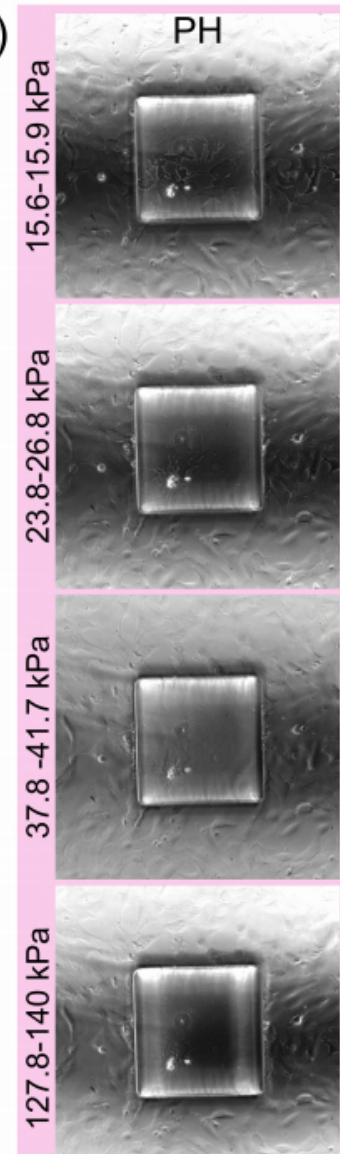
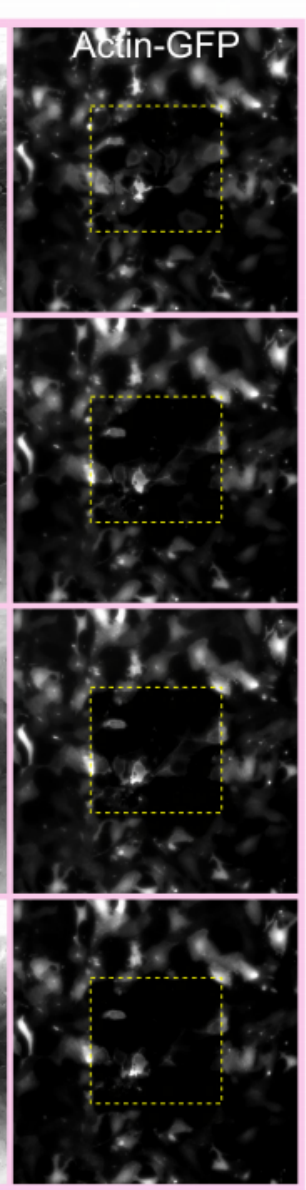

b)
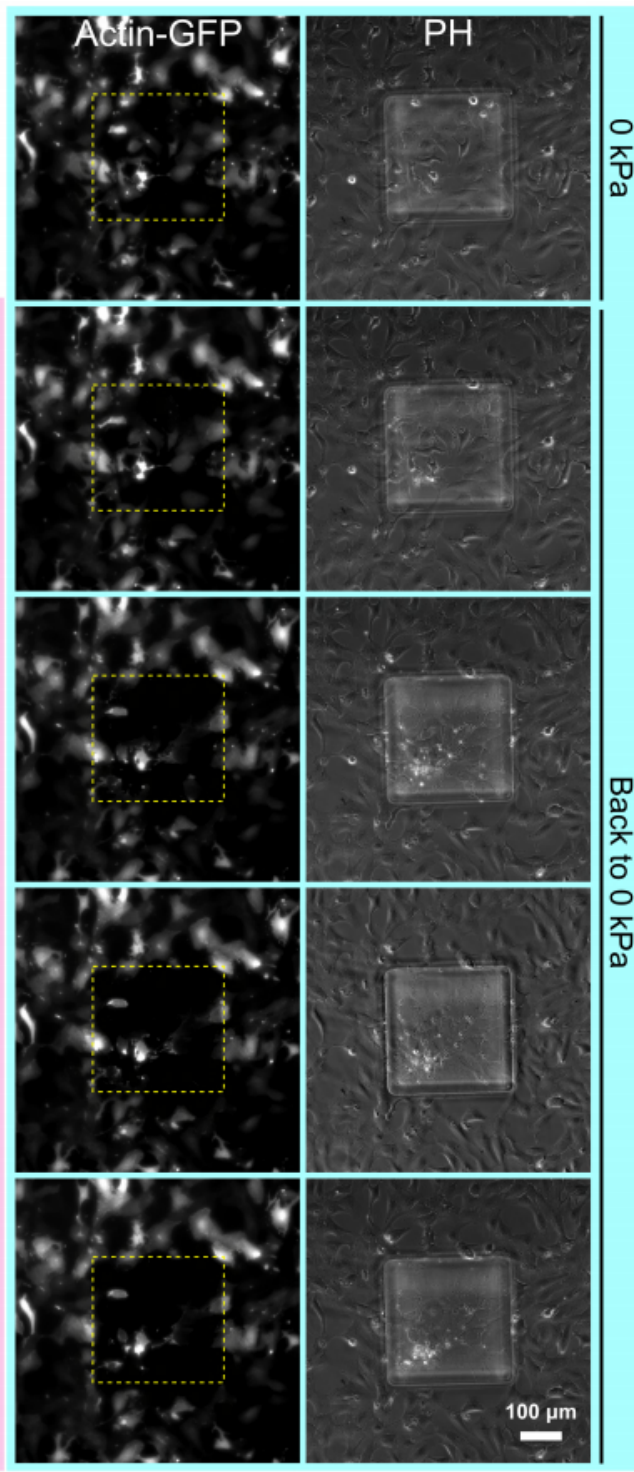

Fig 3. Representative fluorescent microscopy images of live imaging of GFP-tagged actin cytoskeleton of cells. (a) Schematic of the on-chip BacMam transduction into cancer cells for the expression of actin-GFP fusion protein. (b) Phase contrast (right) and fluorescent (left) images of the GFP-tagged actin expressing SKOV-3 ovarian cancer cells at static state $(0 \mathrm{kPa})$ or retracted back to static state $(0$ $\mathrm{kPa}$ ) after compression at the given pressure range. (c) Phase contrast (left) and fluorescent (right) images showing the change in GFP-tagged actin in the cells at the compressed state by the applied pressure. The pressure was applied sequentially in ascending order.

was observed between the control and compressed cells at $15.6 \mathrm{kPa}$ for areal and axial cell nuclei deformation, while compressed cells had larger nuclei than $24 \mathrm{~h}$-recovered compressed cells (Fig $5(\mathrm{~b}, \mathrm{~d})$ ). Conversely, circularity of the cell nuclei was significantly reduced in the compressed cells compared to the control (non-compressed) at zero time after compression, while not significant in $24 \mathrm{~h}$-recovered compressed group compared to control (Fig 5(c)). Differences between the compressed cells fixed at zero time or after 


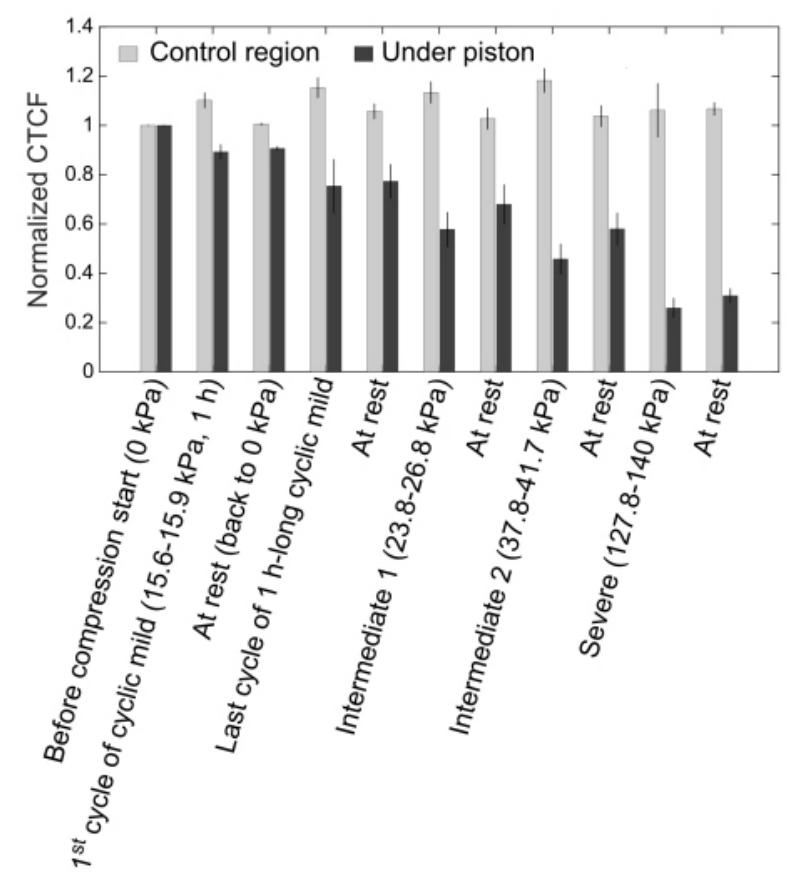

Fig 4. Corrected total cell fluorescence (CTCF) calculated from images of GFP-tagged actin cytoskeleton of cells during sequential cyclic compression at the indicated pressures. Results represent at least 3 independent experiments.

24 h-recovery suggest that cells exhibit deformations at the time of the compression, a proposed mechanism cells use to prevent mechanical damage 1, 17]. These deformations were not permanent morphologically and could be recovered at mild pressures, such as $15.6 \mathrm{kPa}$. No statistical difference between nuclei circularity of the $24 \mathrm{~h}$-recovered compressed cells under micro-piston and of the non-compressed cells in the control region within those recovery samples (Fig 5 (c)) shows that 24 h-recovered compressed cells started to behave like the non-compressed cells during the recovery after compression at $15.6 \mathrm{kPa}$. Nonetheless, no statistical difference in nuclei circularity between the zero-time and $24 \mathrm{~h}$-recovered cell groups after compression suggests the existence of cells showing no full recovery. This divergence shows that heterogeneity exists in cancer cell response to mild compression. Although the developed platform is not a single-cell compression platform, cells could be assessed as single cells for their nuclei despite overlapping cellular parts due to dense culture formation on Matrigel coatings. These experiments enabled to determine cell response to lower physiological compression values, such as $15.6 \mathrm{kPa}$ applied in a cyclic manner for 1 hour. To further understand whether cells exhibit a different cell recovery profile after compression, it was needed to test an upper physiological value.

Utilising the full pressure range capabilities of the platform, we also investigated cell recovery after compression at upper physiological values, such as $20.8 \mathrm{kPa}$ (S2 Fig). For this, cells that were compressed at upper mild pressures were assessed for zero time and 24 h-recovery after the compression and compared to non-compressed control cells in each group (Fig 6 and S5 Fig). Cells developed distinct actin deformation, as shown by the increased fluorescence signals at the edges of the compressed cells under the micro-piston at zero time after cyclic compression at $20.8 \mathrm{kPa}$ for 1 hour, with some cells having their actin disrupted over the nuclei and cytoplasm while highly increased stress fibers presented at the cell membrane (Fig 6(a)). This result is consistent with 
bioRxiv preprint doi: https://doi.org/10.1101/2022.01.07.475458; this version posted January 10, 2022. The copyright holder for this preprint (which was not certified by peer review) is the author/funder. All rights reserved. No reuse allowed without permission.

a)
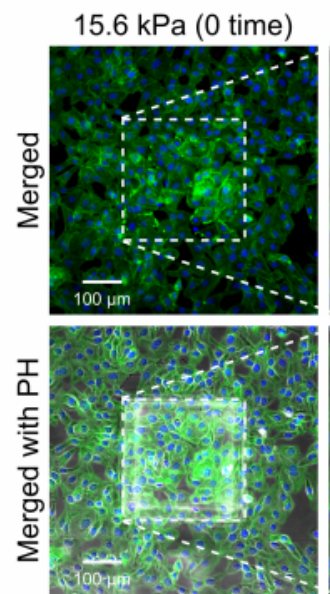

b)

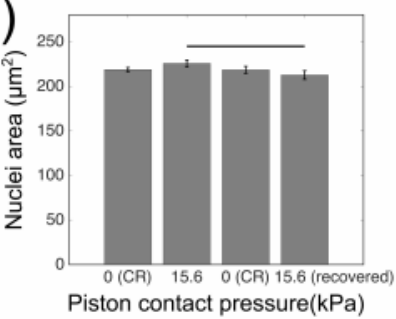

Magnified
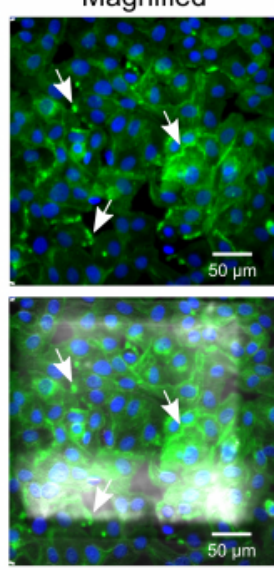

c)

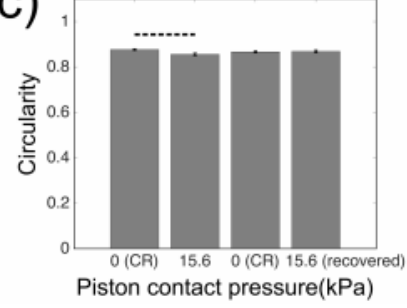

$15.6 \mathrm{kPa}$ (recovered)

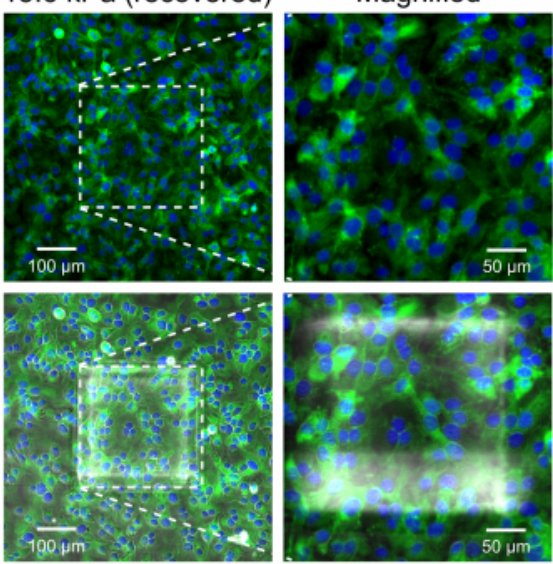

d)

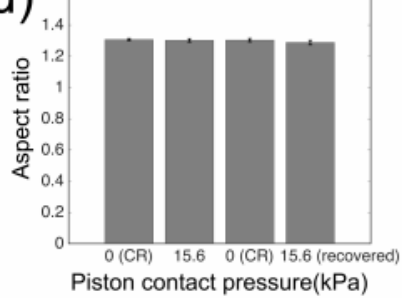

Fig 5. End point assay staining and analysis for actin and nuclei of cancer cells fixed at zero time or after 24 h-recovery, following 1 hour-long cyclic compression between 0 and $15.6 \mathrm{kPa}$. (a) Representative images of control and compressed cell groups stained for actin (green) and nuclei (blue) for their form at zero time and 24 h-recovery after cyclic compression. Dashed areas are under piston, while the surrounding is control region (CR). Merged: merged form of the phalloidin (stain for actin) and Hoechst (stain for nuclei) epi-fluorescence images; merged with $\mathrm{PH}$ : merged form of the epi-fluorescence images with the corresponding phase-contrast $(\mathrm{PH})$ image; magnified: two-hundred-fold magnification images of the region under micro-piston. Representative arrows (white) show the distinct actin deformation by the increased fluorescence signals at the edges of the cells in the compressed groups under the micro-piston at zero time after compression. Nuclei deformation in control cells at control regions (CR) versus in compressed cells under piston at zero time or 24 h-recovery after cyclic compression, measured for area (b), circularity (c) and aspect ratio (d) of the nuclei (mean \pm s.e.m. $\mathrm{n}=588,230,358,174$ cell nuclei). Dashed horizontal bars show significant differences between the control region (CR) group and compressed group under micro-piston. Continuous horizontal bars show the comparison pairs between the cell groups under the micro-piston at zero time and 24-recovery after compression.

changes in actin cytoskeleton and formation of cell surface projections in compressed peritoneal mesothelial cells in the study by Asem et al. 9]. Interestingly, cell actin signal was localized mostly in compressed cells under micro-piston at 24 h-recovery after cyclic compression (Fig 6(a)), compared to control cells (S5 Fig). For compressed cells, this outcome indicates no full recovery from impact of the compression at $20.8 \mathrm{kPa}$, and thus an altered actin cytoskeleton profile. Shape descriptors of the cell nuclei were measured for zero-time and $24 \mathrm{~h}$-recovery after the cyclic compression. There was no statistical difference in nuclei area between compressed or $24 \mathrm{~h}$-recovered compressed groups and with their control groups (Fig 6(b)). On the other hand, circularity of the 
compressed and 24 h-recovered compressed cells was statistically different both between each and respective control groups (Fig 6(c)). Furthermore, aspect ratio of the 24 h-recovered compressed cell nuclei was statistically significantly higher than its 24 h-recovered control group and compressed group at zero time after compression (Fig 6(d)). These results suggest that cells did not fully recover after cyclic compression at upper mild physiological pressure values such as $20.8 \mathrm{kPa}$. The phenotypical response of compressed cells during recovery after compression at $20.8 \mathrm{kPa}$ differed observably from that for $15.6 \mathrm{kPa}$, demonstrating the capability of the flexible microdevice to capture minute differences in cell behaviour after being compressed at various pressures in physiologically relevant manner. Changes in cell actin and nuclei shape during recovery after upper mild compression may indicate formation of more aggressive and metastatic cells [18].

In the study by Ho et al., the height of breast epithelial cells recovered fully after 6 minutes of cyclic compression applied between the external pressures of 10 and 15 psi at $0.5 \mathrm{~Hz}[2]$. No statistical difference in cell height before and after compression was observed, indicating that the cells did not exhibit permanent plastic deformation. Measurement of the viscoelastic relaxation of the cells is difficult however, especially when vertical and temporal resolution in imaging are limited, and long image acquisition times required for 3D visualization might prevent capturing the changes in cell height at the time of compression. In the current study, we thus deliberately limited cell deformation assessment to planar dimensions including area, circularity, and aspect ratio [12, as wide-field imaging is fast enough (e.g. milliseconds long) to capture cell states along the steps of the compression process compared to real-time volumetric imaging (e.g. minutes long) using confocal microscopy [2,5, $5,22$.

Cyclic compression in the study of Ho et al. was applied only for 6 minutes in total, significantly shorter than the entire duration of a cyclic compression in this work, and current results suggest that cell recovery after compression depends on the temporal length of the compression on cells. The overall extent of recovery of compressed cells represented by cell membrane bulges, the actin cytoskeleton and measurements of shape descriptors of cell nuclei can yield further insight into the plasticity of the cancer cells. Observable from S1 Movie, cell plasticity depends on the amount of pressure applied, as well as the timing. Cell bulge formation slowly developed during the compression stage while cells were responding to contact pressure at the interface with the PDMS piston. This was despite the fact that the piston was actuated onto the cells rapidly as directed by the external pressure controller. As shown by the pressure sensor readings in S1 Movie, PDMS membrane and piston were persistently responding to amount, duration and mode of the applied pressure. Thus, the gradual deformation of cells and bulge formation during compression stages must come from response type of the cells to mechanical load at solid contact with the PDMS piston. During rest stages, where the compression was lifted, cell bulges did not fully recover right after compression was removed, suggesting that the cells require a certain amount of time to recover compressed cellular parts S1 Movie). Based on observations of temporal evolution of cell compression application and comparison of application durations of this study with the study by Ho et al. [2], we hypothesize that this recovery also depends on the duration and number of the cycles. Our method that is established to apply sequential cyclic compressions and allow cell recovery after compression can be further employed to study effects of compression and decompression in intratumor vessels or endothelial cells to understand structure and functionality of the decompressed vessels, as its importance was shown in a study by Padera et al 23 .

In summary, our platform can control the strength and duration of cyclic compression, while enabling the observation of morphological, cytoskeletal and nuclear changes in cells, thus providing a powerful new tool for the study of mechanobiological 
bioRxiv preprint doi: https://doi.org/10.1101/2022.01.07.475458; this version posted January 10, 2022. The copyright holder for this preprint (which was not certified by peer review) is the author/funder. All rights reserved. No reuse allowed without permission.

a)
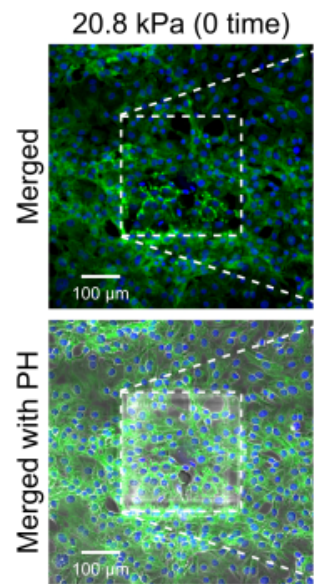

b)

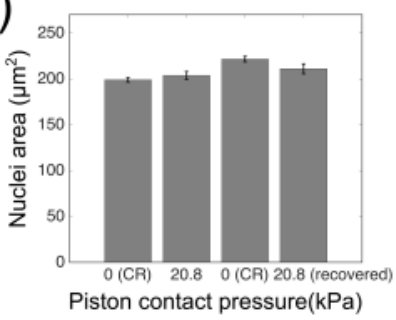

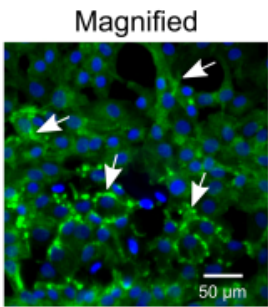

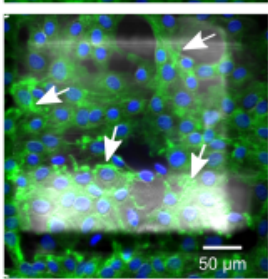

c)

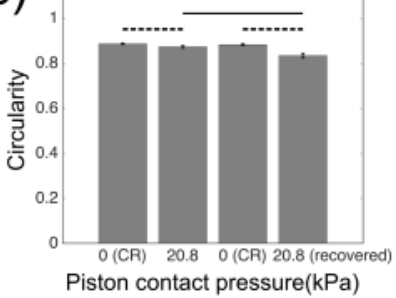

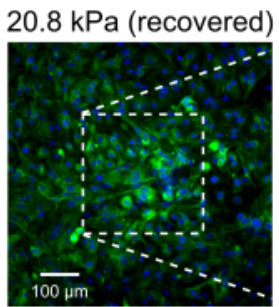

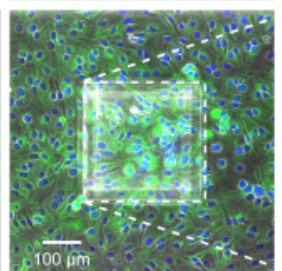

d)

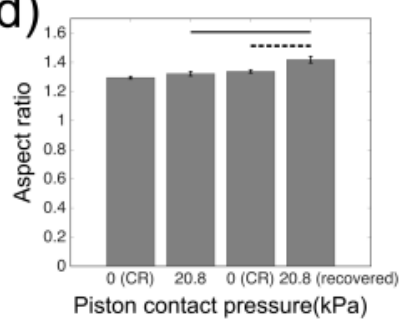

Fig 6. End point assay staining and analysis for actin and nuclei of cancer cells fixed at zero time or after $24 \mathrm{~h}$-recovery, following 1 hour-long cyclic compression between 0 and $20.8 \mathrm{kPa}$. (a) Control and compressed cell groups stained for actin (green) and nuclei (blue) for their form at zero time and 24 h-recovery after cyclic compression. Dashed areas are under piston, while the surrounding is control region (CR). Merged: merged form of the phalloidin (stain for actin) and Hoechst (stain for nuclei) epi-fluorescence images; merged with $\mathrm{PH}$ : merged form of the epi-fluorescence images with the corresponding phase-contrast $(\mathrm{PH})$ image; magnified: two-hundred-fold magnification images of the region under micro-piston. Representative arrows (white) show the distinct actin deformation by the increased fluorescence signals at the edges of the cells in the compressed groups under the micro-piston at zero time after compression where some cells have their actin disrupted over the nuclei and cytoplasm, while highly increased stress fibers at the membrane. Nuclei deformation in control cells at control regions (CR) versus in compressed cells under piston at zero time or 24 h-recovery after cyclic compression, measured for area (b), circularity (c) and aspect ratio (d) of the nuclei (mean \pm s.e.m. $\mathrm{n}=406,162,342,143$ cell nuclei). Dashed horizontal bars show significant differences between the control region (CR) group and compressed group under micro-piston. Continuous horizontal bars show the comparison pairs between the cell groups under the micro-piston at zero time and 24-recovery after compression.

processes in cancer and cell biology. Furthermore, the data obtained in this work suggest that cancer cells showed a plastic cell response to applied sequential cyclic compression. Cells could do that by deformations and perturbations in actin cytoskeleton and nuclei. It is proposed that cells exhibit such deformations at the time of compression to compensate for the applied compressive stress and protect themselves against mechanical damage. Those deformations were recovered fully or partially depending on the amount of applied pressure. At upper mild pressures, such as 20.8 $\mathrm{kPa}$, cells developed and used an adaptive mechanism by incomplete shape recovery. Whereas recovery after mechanical loading is important to maintain cell integrity, an 
adaptive mechanism by incomplete shape recovery might lead to more aggressive and metastatic cancer cells.

\section{Conclusion}

We have demonstrated coating of cell culture chambers in micro-piston devices with hydrogels and successful culture of SKOV-3 ovarian cancer cells therein, mimicking the in vivo cellular microenvironment. The devices were then used to cyclically compress cells with controlled micro-piston contact pressures, verified using FEM simulations to account for possible membrane thickness variations. Computational results showed good agreement with experimentally applied cyclic compressions. Live cells were imaged in the micro-piston devices and mild compression was applied to mechanically stimulate and record changes in actin profile dynamics. This was extended to dynamic cyclic compressions and recording of the long-term cellular changes in mechanosensitive subcellular structures, such as actin cytoskeleton and nuclei, while allowing cells to recover for 24 hours after compression. Details of morphological cell deformations during sequential cyclic compression were captured via high-speed imaging. Membrane bulges in cells fixed directly after compression appeared as increased actin signal at cell edges. We also conclude that recovery of the compressed live cells were depending on magnitude of the applied compression. As demonstrated here with controlled micro-scale mechanical cell compression in a flexible microdevice, our comprehensive method will provide a more accurate replication of cell-physiological mechanisms to study both short- and long-term effects of compression in cellular microenvironments.

\section{Supporting information}

S1 Fig. On-chip growth of SKOV-3 cells on Matrigel-coated glass surface in micro-piston device. Representative images of two samples during culture from zero time to Day 4 after piston-retracted loading of cells at -615 mbar.

S2 Fig. Examples of 24 h-recovery of mildly and upper-mildly compressed SKOV-3 cells on Matrigel-coated glass surface in micro-piston device.

S3 Fig. Image analysis of nuclear shape after cell compression on Matrigel-coated surfaces. Regions of interest (ROIs) extracted for the cell nuclear boundaries in Hoechst (stain for nuclei) epi-fluorescence channel (blue in merged/composite frames) were used to measure area, circularity, and aspect ratio of the cell nuclei, as independent of phalloidin (stain for actin) epi-fluorescence channel (green in merged/composite frames). Merged - 200X: merged form of the phalloidin and Hoechst epi-fluorescence images obtained at two-hundred-fold magnification; merged with PH - 200X: merged form of two-hundred-fold magnification epi-fluorescence images with the corresponding phase-contrast $(\mathrm{PH})$ image for the region under micro-piston; merged with PH - 100X: merged form of one-hundred-fold magnification epi-fluorescence images with the corresponding phase-contrast $(\mathrm{PH})$ image for the region under and around micro-piston in channel.

S4 Fig. End point assay staining and analysis for actin and nuclei of cancer cells in control region (CR) fixed at zero time or after 24 h-recovery, following 1 hour-long cyclic compression between 0 and $15.6 \mathrm{kPa}$. Control cell groups stained for actin (green) and nuclei (blue) for their form at zero time and 24 h-recovery. Merged: merged form of the phalloidin (stain for actin) and Hoechst (stain 
for nuclei) epi-fluorescence images; merged with $\mathrm{PH}$ : merged form of the epi-fluorescence images with the corresponding phase-contrast $(\mathrm{PH})$ image; Random region 200X: two-hundred-fold magnification images of control cells in random region as part of the control region.

S5 Fig. End point assay staining and analysis for actin and nuclei of cancer cells in control region (CR) fixed at zero time or after $24 \mathrm{~h}$-recovery, following 1 hour-long cyclic compression between 0 and $20.8 \mathrm{kPa}$. Control cell groups stained for actin (green) and nuclei (blue) for their form at zero time and 24 h-recovery. Merged: merged form of the phalloidin (stain for actin) and Hoechst (stain for nuclei) epi-fluorescence images; merged with $\mathrm{PH}$ : merged form of the epi-fluorescence images with the corresponding phase-contrast $(\mathrm{PH})$ image; Random region 200X: two-hundred-fold magnification images of control cells in random region as part of the control region.

S1 Movie. Temporal evolution of the dynamic cell compression within Matrigel-coated micro-piston devices. Time-lapse live cell imaging with $100 \mathrm{~ms}$ per frame was recorded for ladder pressure increase from $0 \mathrm{kPa}$ up to $15.6 \mathrm{kPa}$ and a short cycle at $15.6 \mathrm{kPa}$ with gradual increase (from $0 \mathrm{kPa}$ to $15.6 \mathrm{kPa}$ ) and decrease (from $15.6 \mathrm{kPa}$ to $0 \mathrm{kPa}$ ) in $30 \mathrm{~s}$ for each. These steps were sequentially followed by 1 h-long cyclic compression between $0 \mathrm{kPa}$ and $15.6 \mathrm{kPa}$ (piston contact pressure from simulation). Cells were compressed in the temporal evolution of the dynamic pressure control within Matrigel-coated micro-piston devices and dynamic changes in cells during the cyclic compression were captured in detail at a rate of $100 \mathrm{~ms}$ per frame.

S2 Movie. Representative data recording of the dynamic changes of GFP-tagged live cell actin during application of sequential cyclic compression at various applied pressures. SKOV-3 ovarian cancer cells were transduced on-chip with CellLight Actin-GFP, BacMam 2.0 baculovirus (Invitrogen) to study the dynamic biomechanical processes under compression. The actin cytoskeleton profile was quantified for the cancer cell response under applied forces at the compressed stages and at rest stages during the compression process.

S1 File. Corrected total cell fluorescence (CTCF) data for actin integrated density and statistical test results.

\section{Acknowledgments}

We thank Helen Devereux, Gary Turner, and Nicole Lauren-Manuera for assistance. We also thank Mathieu Sellier for help with COMSOL, and Campbell Sheen for assistance with the EPA protocol to use the Actin-GFP BacMam construct. Financial support was provided by the MacDiarmid Institute for Advanced Materials and Nanotechnology and the Biomolecular Interaction Centre.

\section{Competing interests}

The authors have declared that no competing interests exist. 


\section{References}

1. Bonakdar N, Gerum R, Kuhn M, Spörrer M, Lippert A, Schneider W, et al. Mechanical plasticity of cells. Nature materials. 2016;15(10):1090-1094.

2. Ho KK, Wang YL, Wu J, Liu A. Advanced microfluidic device designed for cyclic compression of single adherent cells. Frontiers in Bioengineering and Biotechnology. 2018;6:148. doi:10.3389/fbioe.2018.00148.

3. Novak CM, Horst EN, Lin E, Mehta G. Compressive Stimulation Enhances Ovarian Cancer Proliferation, Invasion, Chemoresistance, and Mechanotransduction via CDC42 in a 3D Bioreactor. Cancers. 2020;12(6):1521. doi:10.3390/cancers12061521.

4. Onal S, Alkaisi MM, Nock V. A microfluidic platform for applying localized and dynamically-controlled compression on cancer cells. In: Proceedings of the 23rd International Conference on Miniaturized Systems for Chemistry and Life Sciences (MicroTAS 2019); 2019. p. 638-639.

5. Onal S, Alkaisi MM, Nock V. A Flexible Microdevice for Mechanical Cell Stimulation and Compression in Microfluidic Settings. Frontiers in Physics. 2021;9:280. doi:10.3389/fphy.2021.654918.

6. Bregenzer ME, Horst EN, Mehta P, Novak CM, Repetto T, Mehta G. The Role of Cancer Stem Cells and Mechanical Forces in Ovarian Cancer Metastasis. Cancers. 2019;11(7):1008. doi:10.3390/cancers11071008.

7. Jain RK, Martin JD, Stylianopoulos T. The role of mechanical forces in tumor growth and therapy. Annual Review of Biomedical Engineering. 2014;16:321-346. doi:10.1146/annurev-bioeng-071813-105259.

8. Fabry B, Maksym GN, Butler JP, Glogauer M, Navajas D, Fredberg JJ. Scaling the microrheology of living cells. Physical review letters. 2001;87(14):148102.

9. Asem M, Young A, Oyama C, ClaureDeLaZerda A, Liu Y, Ravosa MJ, et al. Ascites-induced compression alters the peritoneal microenvironment and promotes metastatic success in ovarian cancer. Scientific Reports. 2020;10(1):1-14. doi:10.1038/s41598-020-68639-2.

10. Nia HT, Munn LL, Jain RK. Physical traits of cancer. Science. 2020;370(6516):eaaz0868.

11. Onal S, Turker-Burhan M, Bati-Ayaz G, Yanik H, Pesen-Okvur D. Breast cancer cells and macrophages in a paracrine-juxtacrine loop. Biomaterials. $2021 ; 267: 120412$.

12. Schindelin J, Arganda-Carreras I, Frise E, Kaynig V, Longair M, Pietzsch T, et al. Fiji: an open-source platform for biological-image analysis. Nature Methods. 2012;9(7):676-682. doi:10.1038/nmeth.2019.

13. Fitzpatrick M. Measuring cell fluorescence using ImageJ. The Open Lab Book. $2014 ;$

14. Shieh AC. Biomechanical forces shape the tumor microenvironment. Annals of Biomedical Engineering. 2011;39(5):1379-89. doi:10.1007/s10439-011-0252-2. 
15. Onal S, Alkaisi M, Nock V. Sequential cyclic compression profiles applied on cancer cells in a flexible microdevice. In: Proceedings of the 47th Micro and Nano Engineering Conference (MNE 2021); 2021.

16. Takao S, Taya M, Chiew C. Mechanical stress-induced cell death in breast cancer cells. Biology Open. 2019;8(8):bio043133. doi:10.1242/bio.043133.

17. Nava MM, Miroshnikova YA, Biggs LC, Whitefield DB, Metge F, Boucas J, et al. Heterochromatin-driven nuclear softening protects the genome against mechanical stress-induced damage. Cell. 2020;181(4):800-817.

18. Lyons SM, Alizadeh E, Mannheimer J, Schuamberg K, Castle J, Schroder B, et al. Changes in cell shape are correlated with metastatic potential in murine and human osteosarcomas. Biology open. 2016;5(3):289-299.

19. Kim YC, Kang JH, Park SJ, Yoon ES, Park JK. Microfluidic biomechanical device for compressive cell stimulation and lysis. Sensors and Actuators B: Chemical. 2007;128(1):108-116. doi:10.1016/j.snb.2007.05.050.

20. Hosmane S, Fournier A, Wright R, Rajbhandari L, Siddique R, Yang IH, et al. Valve-based microfluidic compression platform: single axon injury and regrowth. Lab on a Chip. 2011;11(22):3888-95. doi:10.1039/c1lc20549h.

21. Lee D, Erickson A, You T, Dudley AT, Ryu S. Pneumatic microfluidic cell compression device for high-throughput study of chondrocyte mechanobiology. Lab on a Chip. 2018;18(14):2077-2086. doi:10.1039/C8LC00320C.

22. Paggi CA, Venzac B, Karperien M, Leijten JCH, Le Gac S. Monolithic microfluidic platform for exerting gradients of compression on cell-laden hydrogels, and application to a model of the articular cartilage. Sensors and Actuators B: Chemical. 2020;315:127917. doi:10.1016/j.snb.2020.127917.

23. Padera TP, Stoll BR, Tooredman JB, Capen D, di Tomaso E, Jain RK. Cancer cells compress intratumour vessels. Nature. 2004;427(6976):695-695. doi:10.1038/427695a. 